\title{
The cost-benefit analysis of a coronary care unit
}

\author{
P. C. Reynell and M. C. Reynell \\ From the Bradford Royal Infirmary
}

' A five-bedded coronary care unit located in a general medical ward in a district general hospital has been subjected to a cost-benefit analysis and the results expressed in cost per life saved, assuming that the only benefit of the unit was resuscitation from cardiac arrest with survival to leave hospital. Direct costs were $£ 320$ per life saved. Indirect costs were also incurred because the existence of the unit resulted in the admission of patients who would otherwise have been treated at home, though this was mitigated by a reduction in mean length of stay during the first five years of the unit's existence. Net indirect costs were £220 per life saved, giving a total cost of £540 per life saved. It is suggested that this kind of outcome analysis by survival could be used to assess the performance of other types of coronary care unit and of other forms of expensive medical treatment.

In comparing one type of medical care with another and assessing priorities within the Health Service, it is desirable to have some measure of cost-effectiveness. Attempts to apply the techniques of cost-benefit analysis to the medical services may fail because of the difficulty of expressing benefits in financial terms. There are, however, certain situations in medicine in which it is possible to analyse outcome in terms of lives saved, and the coronary care unit is one of these.

We have therefore carried out a cost-benefit analysis of the work of a coronary care unit using cost per life saved as a measure of effectiveness.

The five-bedded unit sited in a general medical ward of a district general hospital has been described previously (Reynell, I969). No major structural alterations were required.

The unit was designed to economize as far as possible in the use of scarce nursing staff and does not normally admit patients over 65 years old. Patients normally spend three days on the

the coronary care unit and are then transferred to a general medical bed. There were 120 general medical beds in the hospital. One was s sacrificed to make space for resuscitation equipment, but otherwise the number of beds has remained constant throughout the period $\rightarrow$ under consideration.

\section{Direct costs (Table I)}

In these inflationary times, we should point out that costs refer throughout to those prevailing in 1970.

Received 9 December 1971.
Salaries In most coronary care units salaries are by far the largest single item of cost. Doctors and nurses are resources which are not only expensive but in short supply, and this was the main reason which prompted us to establish a unit within a general medical ward. In theory no extra doctors, nurses, or technicians were employed to staff the unit, but in practice the nursing complement was usually a little higher than that of a comparable male medical ward without a coronary care unit. Over the year the average difference was approximately one staff nurse during the day and one second-year nurse at night with replacements for sickness and holidays. We have taken this into account in assessing costs. The coronary care unit undoubtedly involved an increase in work-load on the ward, and we believe that this increase amounted to more than the working time of one nurse. This could mean that other patients in the ward were relatively neglected and it might imply a hidden deficit on the benefit side of the cost-benefit equation. We do not believe that this happened, but can produce no objective evidence to support our belief. We consider that the extra work-load was successfully absorbed by the existing medical and nursing staff. In economic terms this means more efficient use of labour. In human terms it means that well-motivated doctors and nurses were willing to work harder.

Drugs, etc We have taken into account drugs, oxygen, infusions, etc. used exclusively for acute coronary care. As these were not always accounted for separately from those used 
TABLE I Direct costs of coronary care unit per annum

\begin{tabular}{lr}
\hline Salaries & $£ 2000$ \\
Drugs, oxygen, infusions, etc. & $£ 700$ \\
Depreciation of capital equipment & $£ 565$ \\
Repairs, etc. & $£ 580$ \\
\hline & $£ 3845$ \\
\hline
\end{tabular}

in the rest of the ward, it was necessary to rely on the ward sister's estimate of consumption.

Depreciation on capital equipment (defibrillators, oscilloscopes, electrocardiograph, pacemaker, mobile suction, anaesthetic trolley, special beds, and wall sphigmomanometers) The capital costs were those prevailing in 1966 and replacement costs would now be somewhat higher. Approximate life spans were estimated for each item and they were assumed to depreciate in a linear rather than an exponential manner.

Repairs These included replacement of leads, disposable electrodes, electrocardiograph paper, and other items of expendable equipment accounted for in the department of cardiology rather than the ward.

\section{Indirect costs (Table 2)}

The establishment of a coronary care unit may involve indirect costs by altering the pattern of delivery of medical care within the catchment area of the hospital. In particular, it is likely to result in the admission of patients who were previously treated at home. The annual admission rates to the unit during the first five years of its existence (Fig.) provide strong presumptive evidence that this was so. The figures suggest that it was over a year before there was much change in the referring habits of local practitioners. Admissions then rose steeply for two years, but thereafter appeared to be reaching a plateau. Fortunately this pattern of admission results in an S-shaped curve which makes it easy to estimate that by the 5th year admissions exceeded by about 160 per annum the number which would have occurred if there had been no coronary care unit. This involves the assumption that the admission rate of suspected myocardial infarcts in patients under the age of 65 would have remained constant over the fiveyear period if no coronary care unit had been established.

It is therefore necessary to include relevant in-patient costs for 160 extra patients per
TABLE 2 Indirect costs of coronary care unit per annum

Extra admissions p.a. $\times$ mean length of stay $\times$ in-patient costs per day
$($ I60)
$($ I6.7 days $)$
$(£ 2.50)$
$=£ 6680$ p.a.

Deduct

Reduced length of stay $\times$ annual admissions $\times$ in-patient costs per day (3.I days)

(520)

$(£ 2 \cdot 50)$

$=£ 4030$ p.a.

Total $=£ 2650$ p.a.

annum. Under this heading we include catering, laundry, power-light-heat, medical records, drugs, diagnostic radiology, pathology, and pharmacy. We omitted those items of cost not incurred by these particular patients. Costs were obtained from the weekly itemized costs per hospital in-patient per week provided by the hospital finance department. The in-patient costs as defined were $£ 2.50$ per patient per day. The total cost per annum is calculated by multiplying in-patient costs per day by the number of patients (I60) and by the mean length of hospital stay of all patients admitted to the unit during the fifth year (I6.7 days).

The admission of extra patients led to increased pressure on medical beds and this resulted in a reduction in the mean length of stay of all patients admitted to the unit of 3.I days over the five-year period. We therefore feel entitled to deduct in-patient costs for 3.I days for each patient admitted to the unit in a year. This reduced calculated indirect costs to $£ 2650$ per annum. To the best of our knowledge our patients were none the worse

FIG. The increase in admissions to the coronary care unit during the first five years of its existence. By the fifth year there is an estimated excess of 160 admissions per annum.

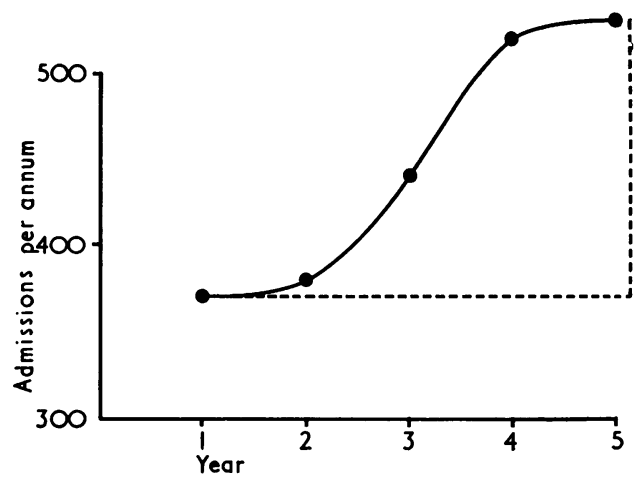


for being discharged earlier. Pressure on beds may also have led to a small decrease in length of stay in the case of some other patients admitted to the general medical wards, but we cannot calculate this with any precision from figures available to us and we believe that any resulting economic benefit was very small.

The establishment of a coronary care unit may have altered the delivery of medical care in the area in other ways, such as increased

' load on ambulance services and decreased load on domiciliary services, but we do not have access to data enabling us to estimate these items and we do not believe that they would amount to any substantial positive or negative net balance.

\section{Benefits}

We propose a new unit of benefit, namely cost per life saved. A better unit would be man/ year/life saved, but this would require reliable survival tables and we do not yet have sufficient data to construct these with any accuracy.

We have assumed that the only benefit of a coronary care unit is resuscitation from cardiac arrest with survival to leave hospital. Some 3 per cent of patients with proven myocardial infarction (WHO criteria) admitted to our unit suffer a cardiac arrest from which they are resuscitated and survive to leave hospital. This figure almost certainly underestimates the true number of lives saved. Other measures such as the early recognition and treatment of dangerous dysrhythmias and of left ventricular failure, and endocardial pacing of some patients with complete heart block may sometimes be life-saving procedures, but there is no way of proving this. We prefer to take into consideration only what we can measure. The number of sur-

1 vivors from cardiac arrest during the first five years of the unit's existence is shown in Table 3. There is some indication that the number per annum is increasing. This is partly due to the increased number of admissions, but we like to think that it is also due to the encouragement of general practi" tioners to admit these patients early and to streamlining of resuscitation procedures. Of

TABLE 3 Number of patients surviving cardiac arrest to leave hospital during first five years of unit's existence

\begin{tabular}{lllllll}
\hline Year & $I$ & 2 & 3 & 4 & 5 & Total \\
\hline Survivors & 3 & 8 & 5 & 10 & 14 & 40 \\
\hline
\end{tabular}

our 40 survivors, 9 were resuscitated at least once by the emergency resuscitation service while in a general medical bed. However, this service is based on and administered by the coronary care unit and staffed by the same doctors and may be regarded as an extension of the unit. In calculating benefit, we have assessed our current performance as 12 lives saved per annum, the average of the last two years. We thus arrive at a cost of approximately $£ 540$ per life saved (direct costs $£ 320$; indirect costs $£ 220$ ).

The increased accuracy of diagnosis achieved by hospital admission rather than domiciliary care of patients with suspected myocardial infarction may well have influenced time spent off work, return to former employment, etc. Twenty-five per cent of admissions to our unit do not have myocardial infarction or coronary insufficiency. This is presumably a net benefit which cannot easily be expressed in quantitative terms.

\section{Discussion}

We pay lip service to the concept that the individual human life is infinitely precious and that no expense must be spared when life is in jeopardy, but advances in life-saving medical treatment are becoming increasingly costly and it is obvious that infinite financial resources are not likely to be made available. More and more we are likely to be faced with the harsh criteria of cost effectiveness in allocating priorities in medicine.

Previous attempts have been made to apply the technique of cost-benefit analysis to aspects of the Health Service (Brooks, 1969; Fein, 1958; Holtman, 1964; Klarman, 1965; Weisbrod, 196I), but it has always proved difficult to express the benefits of medical care in financial terms. Attempts have been made to express results in terms of working days saved, but what monetary value does one put on the work of a housewife or the survival of a pensioner? The application of strict economic criteria might imply that saving the life of a pensioner is a negative benefit, a judgement to which no doctor would subscribe. Too often one is reduced to putting a price tag on judgements which are essentially social and political (Stringer, 1970).

There are, however, certain situations in medicine in which it is possible to estimate with reasonable accuracy the number of lives saved by expensive forms of medical treatment. We have in mind not only different types of coronary care units, but also intensive care units, renal dialysis, organ transplantation, and certain types of expensive surgery, 
in fact any life-threatening situation in which the survival of treated patients can be compared with that of a comparable group for whom the treatment was not available. Money is not always the most important element of 'cost'. Even at its most mechanized, medicine is a labour-intensive industry and scarcity of skilled manpower is sometimes an even greater limiting factor. One can visualize similar cost/ benefit equations expressed as nurse-hours per life saved where this is appropriate. Our own unit was located in a general medical ward in order to economize in scarce nursing staff rather than to save money, and our results could be expressed as one extra nurse/month per life saved, though it is obvious that the small increase in nursing cover was not the only, or even the principal, factor responsible for the saving of lives.

In an outcome analysis by survival one should ideally take into account the quality and duration of survival. We do not yet have sufficient follow-up data to do this, but our results to date show an 82 per cent one-year survival and a 70 per cent two-year survival among survivors from cardiac arrest. Our experience accords with that of others that most of these patients return to work and that long-term prognosis does not differ significantly from that of all patients discharged from hospital after myocardial infarction (Lawrie, 1969; McNamee et al., 1970). A cost of $£ 540$ per survivor does not appear to be excessive. It may be that more elaborate pur- pose-built and independently staffed coronary care units save more lives at an acceptable extra cost, but there is no evidence that this is so.

\section{References}

Brooks, R. G. (1969). Cost-benefit analysis of patients treated at a rheumatism centre. Annals of the Rheumatic Diseases, 28, 655 .

Fein, R. (1958). Economics of Mental Illness. Basic Books, New York.

Holtman, A. G. (1964). Estimating the demand for public health services: the alcoholism case. Public Finance (The Hague), 19, 351.

Klarman, H. E. (1965). Syphilis control program. In Measuring Benefits of Government Investments, Ed. by R. Dorfman. The Brookings Institution, Washington D.C.

Lawrie, D. M. (1969). Long-term survival after ventricular fibrillation complicating acute myocardial infarction. Lancet, 2, 1085.

McNamee, B. T., Robinson, T. J., Adgey, A. A. J., Scott, M. E., Geddes, J. S., and Pantridge, J. F. (1970). Long-term prognosis following ventricular fibrillation in acute ischaemic heart disease. British Medical fournal, 4, 204.

Reynell, P. C. (1969). Coronary care unit in a general medical ward. British Medical fournal, 2, 502.

Stringer, J. (1970). Cost and benefit assessment in medical care. In Resources in Medicine, p. 23. Ed. by Jane Collins. King Edward's Hospital Fund for London.

Weisbrod, B. A. (I96I). Economics of Public Health. University of Pennsylvania Press, Philadelphia.

Requests for reprints to Dr. P. C. Reynell, Bradfield Royal Infirmary, Duckworth Lane, Bradford 9, Yorkshire. 